\title{
Impact, Impact, Impact
}

mmer wieder werden wir als Herausgeber darauf angesprochen, ob denn das Allergo Journal einen Impact Factor habe oder wann es endlich einen Impact Factor bekommen werde.

Zur Erklärung ein paar Hintergrundinformationen: Der Impact Factor, auf gut Deutsch der Einflussfaktor einer Fachzeitschrift, misst, wie häufig Artikel der besagten Zeitschrift von anderen Zeitschriften in einem gewissen Zeitraum zitiert werden. Je häufiger also Beiträge im Allergo Journal von anderen Wissenschaftlern zitiert werden, desto höher ist der Einflussfaktor des Journals, der heutzutage als Maß für das Ansehen der Zeitschrift betrachtet wird. Besonders im Bereich der akademischen Medizin hat diese „Vermessung der wissenschaftlichen Welt" an Bedeutung gewonnen. Auch wenn der Impact Factor als Maß für wissenschaftliche Qualität längst sehr kritisch hinterfragt wird, scheint er unverändert als Währung für die wissenschaftliche Aktivität von einzelnen Autoren oder ganzen Abteilungen zu dienen - z. B. als Grundlage für die Bewertung von Habilitations- und Berufungsverfahren oder der leistungsorientierten Mittelvergabe. Das Interesse der Autoren am Impact Factor einer Fachzeitschrift ist daher durchaus verständlich, auch wenn man manchmal den Eindruck bekommt, dass bei der Jagd nach Punkten häufig vergessen wird, den eigenen Interessen und Neigungen zu folgen und auch gewagte Fragestellungen zu bearbeiten.

Berechnet wird der Impact Factor vom Institute for Scientific Information (ISI), das als Teil eines Medienkonzerns, der Thomson Reuters Corporation, nahezu ein Monopol auf die „Für das Jahr 2010 ergibt sich für das Allergo Journal ein Scopus Impact Factor von 0,50. Dies ist ein sehr beachtliches Ergebnis. “

Erfassung von Zitationshäufigkeiten besitzt. Voraussetzung für das Erlangen eines Impact Factors ist die Listung einer Fachzeitschrift für den sogenannten ISI Journal Citation Report. Hier ist das Allergo Journal bislang nicht gelistet und folglich wird im ISI Journal Citation Report auch kein Impact Factor berechnet. Das heißt aber nicht, dass das Allergo Journal keinen Impact Factor hat, sondern lediglich, dass es bei Thomson Reuters nicht geführt wird.

Zum Glück gibt es seit 2004 Scopus als eine weitere Zitationsdatenbank, die quasi als Konkurrenzprodukt ebenfalls ermöglicht, einen Impact Factor zu errechen. Für das Jahr 2010 ergibt sich hieraus für das Allergo Journal ein Scopus Impact Factor von 0,50. Dies ist ein sehr beachtliches Ergebnis, da es sich um eine nationale Zeitschrift handelt, die vorwiegend auf Deutsch publiziert. Vor dem Hintergrund dieser erfreulichen Zahl werden die Herausgeber und der Verlag im Jahr 2012

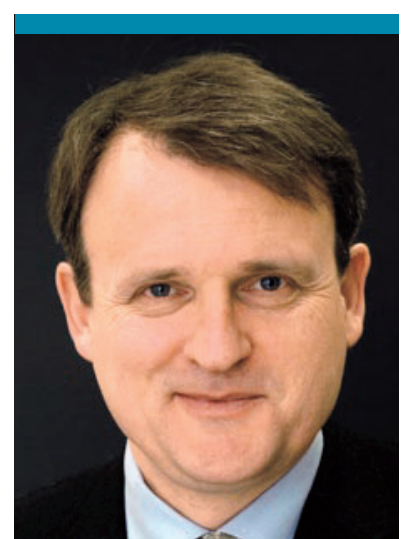

Prof. Dr. Thilo Jakob, Allergieabteilung \& Forschergruppe Allergologie, UniversitätsHautklinik Freiburg

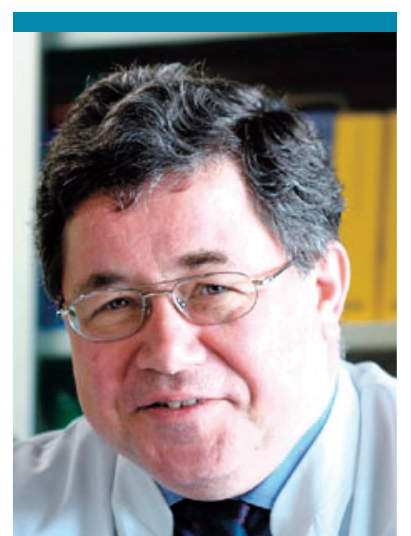

Prof. Dr. Hans F. Merk, Hautklinik der Medizinischen Fakultät, Universitätsklinikum der RWTH Aachen einen erneuten Anlauf nehmen, um das Allergo Journal auch bei Thomson Reuters listen zu lassen.

Unabhängig von Listung und Einflussfaktoren gilt nach wie vor, dass das Allergo Journal mit einer Auflage von 8.000 Exemplaren die allergologische Fachzeitschrift mit der größten Verbreitung im deutschsprachigen Raum ist. Damit dies auch so bleibt, werden wir unsere Sichtbarkeit im Internet verbessern. So wird das Allergo Journal ab dem nächsten Jahr auf den Webseiten von Springer Medizin und Springer Link zu finden sein. Zusätzlich konnten wir den Einreichungs- und Begutachtungsprozess professionalisieren und beschleunigen. Die durchschnittliche Gutachtendauer von Einreichung bis Rückmeldung beim Autor liegt aktuell bei 35 Tagen.

Wir blicken also mit Freude auf eine Vielzahl von Verbesserungen, die das Allergo Journal für Sie als Leser und Autoren noch attraktiver machen werden, als es ohnehin schon ist.

$\mathrm{Zu}$ guter Letzt möchten wir alle unter Ihnen, die an Universitäten oder anderen öffentlichen Institutionen wissenschaftlich tätig sind, auffordern, unbedingt an der Fachgutachterwahl der Deutschen Forschungsgemeinschaft vom 7. November bis 5. Dezember 2011 teilzunehmen, bei der erstmals auch das Gebiet der Allergologie abgebildet ist. Weitere Details finden Sie auf Seite 424.

Viel Freude bei der Lektüre der aktuellen Ausgabe wünschen Ihnen

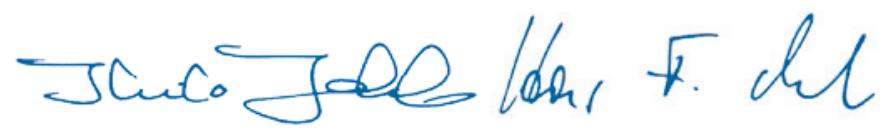

Prof. Dr. Thilo Jakob

Prof. Dr. Hans F. Merk 